\title{
Molecules in the Sun and Molecular Data
}

\author{
Nicolas Grevesse ${ }^{1}$ and A. Jacques Sauval ${ }^{2}$ \\ 1 Institut d'Astrophysique, Université de Liège 5, avenue de Cointe, B-4000 Liège, \\ Belgium \\ 2 Observatoire Royal de Belgique 3, avenue Circulaire, B-1180 Bruxelles, Belgium
}

\section{Introduction}

Molecules play a unique role in solar spectroscopy in allowing to derive, with high accuracy, the temperature structure of the photospheric layers from where the solar spectrum in the near ultraviolet, visible and infrared emerges. They also allow to observe the heterogeneous structure of the outer layers as well as the subtle convective motions in the layers just above the solar convection zone. Molecules play also a unique role in defining the solar chemical composition, at least as far as the very important elements like carbon, nitrogen and oxygen are concerned. They also offer the unique opportunity to derive meaningful isotopic ratios for $\mathrm{C}$ and $\mathrm{O}$. Although we shall not deal with sunspots in the present review, it has to be recalled that molecules are the only tracers to derive the solar abundances of $\mathrm{Cl}$ and $\mathrm{F}$ from the rotation-vibration lines of $\mathrm{HCl}$ and $\mathrm{HF}$ present in the infrared sunspot spectra (Hall \& Noyes 1972; Hall \& Noyes 1969).

On the other hand, the Sun itself offers a unique opportunity to produce molecular transitions, in local thermodynamic equilibrium (LTE), at rather high temperatures $(\mathrm{T} \sim 5000 \mathrm{~K}$ ), higher in any case than in the laboratory, often in non LTE (see review by Johnson in this volume). Therefore, new lines and new transitions of higher excitation appear in the solar spectrum allowing to use the Sun as a "permanent laboratory source" in order to refine the molecular constants. Furthermore, the solar photosphere, where we believe the physical conditions and physical processes are better known than in any other stars, offers a unique possiblity to test basic molecular data like dissociation energies and transition probabilities.

\section{Molecules and the Solar Spectrum}

It is well known that the solar photospheric spectrum, from the near ultraviolet to the infrared, emerges from a very thin layer of about $1 / 1000$ of the solar radius $(700.000 \mathrm{~km})$ just above the solar convection zone. In this region, the photon mean free path becomes rapidly very large whereas it was very small below it. 
Solar spectra of very high resolution and high signal over noise ratio now cover the range from the ultraviolet to the infrared where the most important molecular transitions are found. Let us mention the most important of these solar atlases : Kurucz (1991) and Moore et al. (1982) for references concerning solar atlases, Delbouille et al. (1973; 3000 to $10000 \AA)$, Delbouille et al. $(1981 ; 1$ $\mu \mathrm{m}$ to $5 \mu \mathrm{m})$, Kurucz et al. $(1984 ; 296 \mu \mathrm{m}$ to $1.3 \mu \mathrm{m})$, Blatherwick et al. (1983; IR), Goldman et al. (1987, 1992; IR), Farmer \& Norton (1989; $2 \mu \mathrm{m}$ to $16 \mu \mathrm{m})$, Livingston \& Wallace $(1991 ; 1.1$ to $5.4 \mu \mathrm{m})$, Wallace et al. (1993; 7350 to 11230 $\AA)$, Brekke (1993; 1190 to $1730 \AA$ ). The solar atlas of Farmer \& Norton (1989) is the first infrared solar spectrum obtained from above the earth atmosphere by the ATMOS FTS experiment which flew on board the Spacelab 3 Mission in May 1985. It allows to see, for the first time, the true solar spectrum from 2 to $16 \mu \mathrm{m}$, in a region which is heavily perturbed by telluric absorption.

Table 1. Molecular lines present in the ultraviolet and visible parts of the photospheric spectrum

\begin{tabular}{|c|c|c|c|c|c|}
\hline Molecule & $\begin{array}{c}\text { Electronic } \\
\text { transition }\end{array}$ & $\begin{array}{c}\text { Spectral } \\
\text { region (nm) }\end{array}$ & $\begin{array}{c}\text { Number } \\
\text { of bands }\end{array}$ & $\begin{array}{c}\text { Number } \\
\text { of lines }\end{array}$ & References \\
\hline $\mathrm{H}_{2}$ & $\mathrm{~B}^{1} \Sigma_{u}^{+}-\mathrm{X}^{1} \Sigma_{g}^{+}$ & $120.9-158.1$ & 17 & 77 & $(1)$ \\
$\mathrm{H}_{2}$ & $\mathrm{C}^{1} \Pi_{u}-\mathrm{X}^{1} \Sigma_{g}^{+}$ & $120.9-125.4$ & 2 & 2 & $(2)$ \\
$\mathrm{CO}$ & $\mathrm{A}^{1} \Pi-\mathrm{X}^{1} \Sigma$ & $136.0-167.0$ & 3 & 70 & $(1)$ \\
$\mathrm{SiO}$ & $\mathrm{A}^{1} \Pi-\mathrm{X}^{1} \Sigma$ & $224.0-278.0$ & 3 & 70 & $(3)$ \\
$\mathrm{OH}$ & $\mathrm{A}^{2} \Sigma^{+}-\mathrm{X}^{2} \Pi$ & $281.0-337.8$ & 7 & 457 & $(3)(4)$ \\
$\mathrm{NH}$ & $\mathrm{A}^{3} \Pi-\mathrm{X}^{3} \Sigma^{-}$ & $328.0-348.7$ & 2 & 265 & $(4)$ \\
$\mathrm{CH}$ & $\mathrm{C}^{2} \Sigma^{+}-\mathrm{X}^{2} \Pi$ & $309.1-321.5$ & 2 & 92 & $(4)$ \\
$\mathrm{CH}$ & $\mathrm{B}^{2} \Sigma^{-}-\mathrm{X}^{2} \Pi$ & $362.7-411.9$ & 3 & 142 & $(4)$ \\
$\mathrm{CH}$ & $\mathrm{A}^{2} \Delta-\mathrm{X}^{2} \Pi$ & $413.3-494.1$ & 4 & 486 & $(4)$ \\
$\mathrm{CN}$ & $\mathrm{B}^{2} \Sigma^{+}-\mathrm{X}^{2} \Sigma^{+}$ & $358.3-477.6$ & 9 & 785 & $(4)$ \\
$\mathrm{SiH}$ & $\mathrm{A}^{1} \Pi-\mathrm{X}^{1} \Sigma^{+}$ & $399.2-448.9$ & 2 & 50 & $(5)$ \\
$\mathrm{SiH}$ & $\mathrm{A}^{2} \Delta-\mathrm{X}^{2} \Pi$ & $410.0-419.1$ & 1 & 148 & $(6)$ \\
$\mathrm{CH}$ & $\mathrm{A}^{1} \Pi-\mathrm{X}^{1} \Sigma^{+}$ & $422.3-445.7$ & 1 & 14 & $(7)$ \\
$\mathrm{C}_{2}$ & $\mathrm{~d}^{3} \Pi_{g}-\mathrm{a}^{3} \Pi_{u}$ & $466.4-563.5$ & 8 & 555 & $(4)$ \\
$\mathrm{MgH}$ & $\mathrm{A}^{2} \Pi-\mathrm{X}^{2} \Sigma$ & $495.6-521.0$ & 1 & 114 & $(4)$ \\
$\mathrm{CN}$ & $\mathrm{A}^{2} \Pi-\mathrm{X}^{2} \Sigma^{+}$ & $550.0-2500.0$ & 32 & $>4000$ & $(4)(8-10)$ \\
& & & & & \\
\hline
\end{tabular}

(1) Sandlin et al. (1986), (2) Bartoe et al. (1979), (3) Moore et al. (1982), (4) Moore et al. (1966), (5) Grevesse \& Sauval (1970), (6) Sauval (1969), (7) Grevesse \& Sauval (1971b), (8) Swensson et al. (1970), (9) Sneden \& Lambert (1982), (10) Sauval et al. (1993).

In the solar spectrum, one finds transitions due to diatomic molecules only, made of the most abundant species like $\mathrm{H}, \mathrm{C}, \mathrm{N}$ and $\mathrm{O}$ together with fainter lines 
due to $\mathrm{MgH}, \mathrm{SiH}, \mathrm{SiH}^{+}, \mathrm{CH}^{+}$. Among the transitions, we find the electronic transitions of $\mathrm{CO}, \mathrm{H}_{2}$ and $\mathrm{SiO}$ in the $\mathrm{UV}$, the electronic transitions of $\mathrm{OH}, \mathrm{CH}$, $\mathrm{NH}, \mathrm{C}_{2}$, and $\mathrm{CN}$ and in the infrared, rotation-vibration transitions of $\mathrm{CO}, \mathrm{CH}$, $\mathrm{OH}, \mathrm{NH}$ and pure rotation transitions of $\mathrm{OH}$ and $\mathrm{NH}$. We have to mention here a difficult case of molecular identification: many lines belonging to the blue and green bands of $\mathrm{FeH}$ were tentatively identified in the disk spectrum, essentially based on a statistical test of the wavelength coincidences (Carroll et al. 1976). Later Wing et al. (1977) and Wöhl et al. (1983) showed that there was no evidence for other $\mathrm{FeH}$ bands in the infrared photospheric spectrum (these IR bands are well present in sunspot spectra). Their conclusion is that the blue and green $\mathrm{FeH}$ bands are very probably absent in the photospheric spectrum (see Lambert, 1988, for a review of the FeH identification). Table 1 summarizes the different molecular transitions identified in the ultraviolet and visible parts of the solar photospheric spectrum (see Grevesse \& Sauval, 1991, for a similar table relative to the infrared part of the solar spectrum). Detailed tables of identifications, covering atomic as well as molecular lines, may be found in Moore et al. $(1966 ; 2935$ to $8770 \AA ; 1982 ; 2095$ to $3069 \AA)$, Swensson et al. (1970; 7498 to $12016 \AA)$, Blatherwick et al. (1983; IR), Goldman et al. (1987; IR), Geller (1992, 2 to $16 \mu \mathrm{m})$.

\section{Computation of the Molecular Densities}

Chemical equilibrium is assumed between the different atomic and molecular species. In the solar photosphere where rather few diatomic molecules are formed, the system of equations relating atomic and molecular species is rather simple. Let us write it for $\mathrm{H}, \mathrm{C}, \mathrm{N}$ and $\mathrm{O}$ which produce by far the most abundant diatomic molecules. We shall express it, as usual, in terms of the partial pressures of the different species :

$$
\begin{aligned}
& P_{H}=p_{H}+p_{H+}+p_{H^{-}}+2 p_{H_{2}}+p_{C H}+p_{N H}+p_{O H}+\ldots \\
& P_{C}=p_{C}+p_{C+}+2 p_{C_{2}}+p_{C O}+p_{C N}+p_{C H}+\ldots \\
& P_{N}=p_{N}+p_{N+}+2 p_{N_{2}}+p_{N H}+p_{C N}+\ldots \\
& P_{O}=p_{O}+p_{O+}+2 p_{O_{2}}+p_{C O}+p_{O H}+\ldots
\end{aligned}
$$

where $P_{X}$ is the total pressure of species $X$ and $p_{X}$ is the partial pressure of the sames species under the form indicated (neutral, ionized, molecular). The corresponding number densities, computed from $\mathrm{P}=\mathrm{N} \mathrm{kT}$, will be represented by $\mathrm{N}_{X}$ and $\mathrm{n}_{X}$ respectively.

Other terms and other equations are added in order to include very minor species which are not or hardly seen in the solar spectrum.

The $p_{\boldsymbol{X}}+/ p_{\boldsymbol{X}}$ are computed by the Saha law and the partial molecular pressures obey the Guldberg-Waage law :

$$
p_{A X}=\frac{p_{A} p_{X}}{K_{A X}}
$$


where $K_{A X}$, the so-called equilibrium constant, at temperature $\mathrm{T}$, is given by

$$
K_{A X}=\left(\frac{2 \pi m}{h^{2}}\right)^{3 / 2}(k T)^{5 / 2} \frac{Q_{A} Q_{X}}{Q_{A X}} \exp \left(-D_{0} / k T\right)
$$

The $Q$ 's are the partition functions for the atoms and the molecule; $\mathrm{m}$ is the reduced mass and $D_{0}$ is the dissociation energy of molecule $A X$.

If we express $D_{0}$ in $\mathrm{eV}$, it is convenient to replace exp $\left(-D_{0} / k T\right)$ by $10^{-D_{0} \Theta}$ where $\Theta=5040 / T$. From (2) and (3), we derive easily the number density of molecule $A X$ :

$$
n_{A X}=n_{A} n_{X}\left(\frac{h^{2}}{2 \pi m}\right)^{3 / 2}(k T)^{-3 / 2} \frac{Q_{A X}}{Q_{A} Q_{X}} 10^{D_{0} \Theta}
$$

As in the solar photosphere $T \sim 5000 K, \Theta \sim 1$, we see that an uncertainty of $0.10 \mathrm{eV}$ on the dissociation energy makes the molecular abundance uncertain by 0.10 dex i.e. $25 \%$ ! This problem of the accuracy needed for the dissociation energies is a very serious one. The example of the quite common CN molecule can perhaps best illustrate the point. For long years, the dissociation energy has been very uncertain with values from 7.2 to $7.9 \mathrm{eV}$. Even today, there is still a difference of about 0.12 dex ( $30 \%$ uncertainty on the CN density) between the best experimental value, $7.77 \mathrm{eV}$, and the best theoretical result, $7.65 \mathrm{eV}$ (see review by Costes \& Naulin in this volume and Sect. 8.1).

Another important result is readily derived from relation (4) concerning the temperature dependence of the molecular abundances. Let us take $\mathrm{CO}$ as the best example, with its high dissociation energy, $D_{0}=11.09 \mathrm{eV}$. If we decrease the temperature by $1 \%$ i.e. $50 \mathrm{~K}$ around $5000 \mathrm{~K}$, the number of $\mathrm{CO}$ molecules will increase by $30 \%$ ! $\mathrm{CO}$ is actually the most sensitive temperature indicator (see Sect. 4).

If we know all the equilibrium constants i.e. all the molecular and atomic partition functions and the dissociation energies, the system of equations (1) can easily be solved by an iterative procedure and we thus easily obtain the molecular abundances versus depth in the solar photosphere for a given set of elemental abundances and a given model atmosphere.

Computations of the molecular partition functions and equilibrium constants have been carried out by many authors. Table 2 gives an overview of the most recent investigations on this subject. Detailed discussions on the methods used to calculate exact molecular partition functions have been published, especially for $\mathrm{CO}$ and $\mathrm{H}_{2}$, by Bohn \& Wolf (1984), Sauval \& Tatum (1984; see also Tatum 1988), Chase et al. (1985, JANAF Tables) and Irwin (1987). Most of these determinations lead to polynomial fits as a function of the temperature (or of the reciprocal temperature).

When computing the line opacity of a real molecular line, we need to use the density of molecules in a given excited level. This is computed from the Boltzmann law and this value is inversely proportional to the molecular partition function $Q_{A X}$. When combining this with (4), it is immediately seen, at least for minor species, that the density of a molecule in an excited level is independent of 
Table 2. Partition functions and equilibrium constants for molecules of astrophysical interest

\begin{tabular}{|c|c|c|}
\hline References & Q K & Total number of molecules $(\mathrm{AB}+\mathrm{ABC} .)$. \\
\hline$(1)$ & $\mathrm{K}$ & $232(132+100)$ \\
$(2)$ & $\mathrm{Q}$ & $20(19+1)$ \\
$(3)$ & $\mathrm{Q} \mathrm{K}$ & $>250(100+150)$ \\
$(4)$ & $\mathrm{Q}$ & $66(33+33)$ \\
$(5)$ & $\mathrm{K}$ & $53(26+27)$ \\
$(6)^{(*)}$ & $\mathrm{Q} \mathrm{K}$ & $300(300+0)$ \\
$(7)$ & $\mathrm{Q}$ & $53(26+27)$ \\
$(8)$ & $\mathrm{Q} \mathrm{K}$ & $>500(200+300)$ \\
$(9)$ & $\mathrm{Q}$ & $162(0+162)$ \\
\hline
\end{tabular}

$\left.{ }^{*}\right)$ Note that there is a small error in the tabulated coefficients of log $\mathrm{K}$ relative to 18 of the positive molecular ions, $\mathrm{AB}^{+}$, for which $\mathrm{I}(\mathrm{A})<\mathrm{I}(\mathrm{B})$. The atomic partition functions which were adopted in $K\left(\mathrm{AB}^{+}\right), \mathrm{Q}(\mathrm{A})$ and $\mathrm{Q}\left(\mathrm{B}^{+}\right)$, are incorrect and they have to be replaced by $Q\left(A^{+}\right)$and $Q(B)$ as mentioned in the text.

(1) Tsuji (1973), (2) Scalo \& Ross (1976), (3) Gurvich et al. (1978-1981), (4) Irwin (1981), (5) Rossi \& Maciel (1983), (6) Sauval \& Tatum (1984), (7) Rossi et al. (1985),

(8) Chase et al. (1985, JANAF Tables), (9) Irwin (1988)

the molecular partition function. This is true only if the dissociation constants have been evaluated with the same molecular partition functions!

\section{Molecules and the Temperature Structure}

Empirical models of the solar photosphere are generally built from observations of the absolute solar continuous intensity versus wavelength, from center to limb variation of this intensity and from atomic line profiles. The best photospheric model of Holweger \& Müller (1974) is built in that way; atomic spectral lines allow to extend the model to higher layers because continuum observations of high quality only cover the visible and near infrared and therefore only allow to test the temperature structure in rather deep layers. The model of Holweger \& Müller (1974) is in good agreement with the most recent Harvard-Oslo solar model of Maltby et al. (1986).

We showed how rotation-vibration and pure rotation lines of $\mathrm{OH}$ can be used to test the temperature structure in the solar photosphere (Grevesse et al. 1984; Sauval et al. 1984). This is illustrated in Fig. 5 of Grevesse et al. (1984), where we show the resulting solar abundance of oxygen derived from rotation-vibration and pure rotation lines of different excitation energies. Using 
the Holweger-Müller model leads to the same abundance for all the lines whereas, using the Vernazza et al. (1976) model, an earlier version of the Harvard-Oslo model, leads to a very strong dependence of the derived abundance on the excitation energy. This can only be explained by slight temperature differences between the two models.

We showed in Sect 3. that CO is the most sensitive temperature indicator in the solar photosphere. It is definitely more sensitive than Fe I lines which have been used by different authors (in the photosphere, about $95 \%$ of the $\mathrm{Fe}$ is in the form of $\mathrm{Fe} \mathrm{II} \mathrm{and} 5 \%$ as Fe I).

CO lines have another advantage. With the ATMOS solar spectra from space (Farmer \& Norton 1989) we entirely see all the rotation-vibration lines of the fundamental $(\Delta v=1)$ and first-overtone $(\Delta v=2)$ bands. We can follow the two sequences up to high $v$-values and $J$-values without interruption. This is quite a progress as compared with what can be seen from ground-based spectra. Thus, we have now at our disposal a very large number of $\mathrm{CO}$ lines, from strong to faint lines, which can be measured with very high accuracy (blends are quite rare in these spectral regions). The strongest of these $\mathrm{CO}$ lines are formed very high in the solar photosphere, at about the same geometrical depth as the $\mathrm{Ca}$ II $\mathrm{H}$ and $\mathrm{K}$ lines which indicate the chromospheric temperature rise. The faintest CO lines are formed rather deep. Therefore, $C O$ lines are a unique thermometer which allows to scan layers from above the so-called region of minimum temperature between the photosphere and the chromosphere down to rather deep layers.

We recently reported (Grevesse \& Sauval 1991) how the numerous CO lines in the infrared solar spectrum can be used to refine the photospheric temperature up to very high photospheric layers. Our new empirical model (see Fig. 3 in the hereabove mentioned paper) confirms the role of $\mathrm{CO}$ in the upper photospheric layers.

\section{Line Shifts, Asymmetries and Convection}

Observations of absolute line shifts and asymmetries in the solar line profiles are generally interpreted as the result of the presence of convective motions above the theoretical upper limit of the solar convection zone (see e.g. de Jager 1959). More recently Dravins et al. $(1981,1986)$ and Nadeau (1988) used a large number of Fe I and Fe II lines to make a comprehensive analysis of these shifts and asymmetries.

In a simple qualitative model of the convective motions, hot matter is moving upwards whereas cool matter moves downwards. In such a model, one sees easily when combining the line profiles shifted to the blue (hot matter, higher intensity) and to the red (cool matter, lower intensity), that the resulting line profile becomes asymmetric, each point of the line profile being shifted, relative to its laboratory position, by a different amount.

The numerous CO lines observed in the ATMOS solar spectrum have many advantages when used to study these effects. They are the most sensitive indicators of temperature differences. They are formed in a much wider range of 
depths than the Fe lines previously used. They allow to see what happens to the convective motions when going to extremely high photospheric layers. They do not suffer from hyperfine structure broadening. The isotopic effects are much larger than the line widths. The probability of an unknown blend slightly perturbing the line profile decreases also tremendously as one goes from the visible to the infrared. Finally, the wavenumbers of the CO lines are known with a higher accuracy than the Fe lines in the visible.

We recently showed (Grevesse \& Sauval 1991; Blomme et al. 1993) how the shifts and asymmetries in the CO lines can be used to refine our knowledge of the convective motions over a very wide range of depths.

\section{Solar Abundances of $\mathrm{C}, \mathrm{N}$ and $\mathrm{O}$}

Carbon, nitrogen and oxygen have partially escaped from the meteorites. The solar spectrum is thus the only source of reliable solar system abundances of these important elements. The needs for an accurate knowledge of the solar abundances of these elements have been summarized by Lambert (1978) and Sauval et al. (1984). The most important motivation for refining our knowledge of these values is certainly the fact that they contribute to about $70 \%$ to the metallicity (in the astronomical literature, the metallicity is the sum of the abundances, by mass, of all the elements except for hydrogen and helium). The detailed values of the $\mathrm{C}, \mathrm{N}$ and $\mathrm{O}$ abundances are of crucial importance for the opacity calculations for stellar interiors (OPAL : Iglesias et al. 1992; OPACITY PROJECT : Seaton et al. 1992) as well as for stellar envelopes (Kurucz 1992).

$\mathrm{C}, \mathrm{N}$ and $\mathrm{O}$ have quite a large number of indicators in the solar photospheric spectrum. Lambert (1978) was the first to show the importance of using all the indicators of the abundances of these elements, atoms as well as molecules.

In different papers (Sauval et al. 1984; Grevesse et al. 1984; Grevesse et al. 1990, 1991; Geller et al. 1991; Grevesse \& Sauval 1991; Grevesse et al. 1992; Grevesse et al. 1993) we report the detailed results of new analyses. During these works, it became clear that permitted atomic lines of C I, N I and O I are probably not the best abundance indicators because of blending problems, problems with the transition probabilities (although much progress has recently been made : Biémont et al. 1991; Hibbert et al. 1991; Hibbert et al. 1993) and possible departures from LTE in O I (Kiselman 1991; Grevesse \& Noels 1993). It also became clear that the best abundance indicators are the molecular transitions, i.e. $\mathrm{C}_{2}$ (Swan, Phillips), $\mathrm{CH}(\mathrm{A}-\mathrm{X}), \mathrm{CH}$ rotation-vibration, $\mathrm{NH}$ and $\mathrm{OH}$ rotationvibration and pure rotation. Among these molecular indicators, the best ones are without any doubt the infrared transitions which are less perturbed than the transitions at shorter wavelengths and which can now be measured with high accuracy on the ATMOS solar infrared spectra from space (Farmer \& Norton 1989).

Our analyses have shown that the results from the different indicators agree remarkably. The results have been slightly revised recently (Grevesse et al. 1993) in order to take into account slight changes in the model atmosphere due to a 
reduction in the solar abundance of iron which is a substantial electron donor. The values we recommend are $\mathbf{A}_{C}=8.55, \mathbf{A}_{N}=7.97, \mathrm{~A}_{O}=8.87$, where $\mathbf{A}_{X}$ means $\log \mathrm{N}_{X} / \mathrm{N}_{H}$, in the usual scale where $\log \mathrm{N}_{H}=12$, with an uncertainty of about 0.05 dex.

It is well known that isotopic effects on atomic lines are extremely small as compared to the thermal widths of the lines in the solar spectrum. The only isotopes for which one has reliable abundance data are ${ }^{12} \mathrm{C} /{ }^{13} \mathrm{C}$ and ${ }^{16} \mathrm{O} /{ }^{18} \mathrm{O} /{ }^{17} \mathrm{O}$. These ratios are derived from the rotation-vibration lines of $\mathrm{CO}(\Delta v=1)$.

In the ATMOS spectra, quite a large number of ${ }^{13} \mathrm{C}^{16} \mathrm{O}$ lines are available; lines of ${ }^{12} \mathrm{C}^{18} \mathrm{O}$ are easily measurable and, for the first time in the solar photospheric spectrum, we have identified and measured a few lines of ${ }^{12} \mathrm{C}^{17} \mathrm{O}$. The solar isotopic ratios derived from these new measurements agree, within the error bars, with the telluric values (Anders \& Grevesse 1989;12/13 = 89, 16/18 $=499,16 / 17=2625)$. They also agree with values for ${ }^{13} \mathrm{C}$ and ${ }^{18} \mathrm{O}$ obtained by Harris et al. (1987) from CO fundamental bands observed from a balloon.

\section{The Sun as a "Laboratory Source" for Producing Molecular Spectra}

The advantages of the Sun as a permanent source for producing molecular spectra.has been recalled in the introduction. Molecules can survive in the solar atmosphere, under LTE, at rather high temperatures $(\sim 5000 \mathrm{~K})$ whereas they are often destroyed in the laboratory, even at lower temperatures but in non LTE. The Sun thus provides a fairly complete coverage, especially in rotational excitation, reaching higher values than in the laboratory.

We have shown in different papers (Mélen et al. 1989; Farrenq et al. 1991; Grevesse \& Sauval 1992; Sauval et al. 1992; Grevesse et al. 1991; Geller et al. 1991) that infrared solar spectra from space make it possible to see a large number of new high excitation lines and transitions never seen in the laboratory, and we have shown how these new data have been used to derive improved molecular constants for the ground states of $\mathrm{CH}$ and $\mathrm{CO}$.

\section{Testing Molecular Data using Solar Spectroscopy}

Let us recall (Sect. 3) that the uncertainties in the line absorption coefficient of a molecular line essentially depends on the uncertainties of two molecular quantities, the line oscillator strength, $f_{v} \mathrm{~J}$, and the dissociation energy, $D_{0}$, as well as on the uncertainties in the number densities of the relevant atoms, $N_{\boldsymbol{A}}$ and $N_{\boldsymbol{X}}$, in the photospheric layers. As the atomic abundances can be derived, in most of the cases, using other indicators, it is possible to derive, from a comparison of observed and synthetic line profiles, the "solar value" of a missing molecular parameter if the other is known or a relation between the two parameters if they are both uncertain.

This however, builds on the assumption that the physical processes and physical conditions in the solar photosphere are well known. We discussed the physical 
conditions in Sect. 4. A very large number of observations and theoretical studies have accumulated with the years. They all converge to convince solar spectroscopists that the LTE hypothesis works pretty well in most of the cases (see e.g. Hinkle \& Lambert, 1975). We are even tempted to say that the Sun is never "at fault".

We have shown (Grevesse et al. 1991), in the course of our analysis of the solar carbon abundance derived from many different indicators, how the dissociation energy of $\mathrm{C}_{2}$ has been tested and how the transition probabilities of the $\mathrm{C}_{2}$ Swan $(0-0)$ band and the $C_{2}$ Phillips (0-0) and (1-0) bands have been selected among different available values. Similar tests have been made on NH (Grevesse et al. 1990; Geller et al. 1991) and on OH (Sauval et al. 1984; Grevesse et al. 1984). These solar tests of molecular data are summarized in Grevesse \& Sauval (1992).

\subsection{CN red system}

In collaboration with $\mathrm{R}$. Blomme, we have made a new analysis of a large number of lines of this system in the solar photospheric spectrum. Other recent studies of these lines have been made by Sneden \& Lambert (1982); see also Lambert et al (1986), Sinha \& Tripathi (1986). We have been extremely careful in the selection of the lines. This selection was based on the total half-widths of the lines. We also compared the solar spectrum with a predicted $\mathrm{CN}$ spectrum based on Kotlar et al (1980) for the constants and on Jørgensen \& Larsson (1990) for the intensities. With these two criteria we have been able to reject most of the blended CN lines. The molecular data concerning this system are still uncertain to some extent. Recent experimental values of the dissociation energy cluster around $7.75 \mathrm{eV}$ (Eres et al 1984; Wannenmacher et al. 1990; Costes et al. 1990; Huang et al. 1992) with rather small uncertainties ( 0.02 to $0.05 \mathrm{eV})$ whereas theoretical values are generally much lower $(7.65 \mathrm{eV}$ : Bauschlicher et al. 1988; $7.41 \mathrm{eV}:$ Knowles et al. $1988 ; 7.52 \mathrm{eV}:$ Larsson et al. 1983 ; but $8.05 \mathrm{eV}:$ Rao et al. 1982). For the band oscillator strength (0-0 band), we have also a disagreement between theoretical values, which cluster around $2.3510^{-3}$ (Bauschlicher et al. 1988; Knowles et al. 1988; Lavendy et al. 1984; Cartwright \& Hay 1982; but 3.25 $10^{-3}$ : Larsson et al. 1983), and recent experimental values $\left(2.910^{-3}:\right.$ Lu et al. $1992 ; 1.9410^{-3}$ : Davis et al. $1986 ; 3.110^{-3}$ : Taherian \& Slanger 1984; 3.6 $10^{-3}$ : Jackson et al. 1982; Conley et al. 1980). We predicted the solar strengths of a large number of lines of the $0-0$ band using the abundances of $\mathrm{C}$ and $\mathrm{N}$ derived by using other indicators of these abundances (Sect. 6). In order for the predicted equivalent widths to agree with the observed ones, we varied the two "free" parameters, $f_{00}$ and $D_{0}$, which intervene as a product, $f_{00} 10^{D_{0} \Theta}$. With a high value of $f_{00}=3.2510^{-3}$, we derive $D_{0}=7.85 \mathrm{eV}$. With a low $f_{00}=$ $2.3510^{-3}$ we need a much higher $D_{0}=8.0 \mathrm{eV}$. In the course of this analysis, we also noted that the fit between predicted and observed solar strengths becomes difficult as J" increases. This might possibly be caused by some interaction factor which was omitted in the theoretical calculations. As the Sun is so succesfull in testing molecular data for other molecules, why should it fail for CN ? We feel it is urgent to do more work on the $f_{00}$ as well as on the $D_{0}$ of $\mathrm{CN}$. 


\subsection{Transitions with very faint lines: $\mathrm{SiH}, \mathrm{SiH}^{+}$and $\mathrm{CH}^{+}$.}

The electronic transitions from the ground states of $\mathrm{SiH}\left(A^{2} \Delta-X^{2} \Pi\right), \mathrm{SiH}^{+}$ (A $\left.{ }^{1} \Pi-X^{1} \Sigma^{+}\right)$and $\mathrm{CH}^{+}\left(\mathrm{A}^{1} \Pi-X^{1} \sum^{+}\right)$have been identified in the solar spectrum but the lines are very faint and heavily blended. Therefore quantitative results have rather large uncertainties.

$\mathrm{SiH}$ - The solar $f$-value, for the $0-0$ band is slightly smaller, $3-410^{-3}$ (revised value based on earlier solar measurements by Sauval 1969) and $4.510^{-3}$ (Lambert \& Mallia 1970), than the most recent laboratory value, $f_{00}=510^{-3}$ (Carlson et al. 1978).

$\mathrm{SiH}^{+}$- The revised value based on earlier solar measurements (Grevesse $\&$ Sauval $1970,1971 \mathrm{a}), f_{00}=1.310^{-3}$ agrees very well with the most recent laboratory value, $f_{00}=1.410^{-3}$ (Hishikawa \& Karawajczyk, 1993).

$\mathrm{CH}^{+}$- When we derived the solar $f_{00}$-value (Grevesse \& Sauval 1971b), $f_{00}=$ $1.210^{-3}$, it was much smaller than the first experimental value of Smith (1971), $f_{00}=6.810^{-2}$. This has been the origin of a pacific fight between Smith and ourselves. In the meantime, new laboratory measurements and computations have been published which converge to $f_{00}$ between $5.4510^{-3}$ (Larsson \& Siegbahn 1983; theory) and $5.610^{-3}$ (Mahan \& O'Keefe 1981; experiment).

Based on synthetic spectra, we have also slightly revised our solar value to $f_{00}=(2-4) 10^{-3}$, which is in better agreement with other determinations, showing, once more, that the Sun was right!

\section{Conclusions}

We have shown the very important and unique role played by diatomic molecules in solar spectroscopy. They are the best indicators of the solar abundances of carbon, nitrogen and oxygen. They are also, and especially $\mathrm{CO}$, the most sensitive thermometers which make it possible to scan a wide range of layers in order to derive the temperature in these layers. They allow us to study the heterogeneous structure of the photosphere over a much wider range of optical depth, than previously possible, reaching, for the first time (through the strong $\mathrm{CO}$ lines of the $\Delta v=1$ sequence), very high layers.

The Sun produces molecular transitions at temperatures higher than in the laboratory. The newly observed solar lines are used to refine the molecular constants of some ground states $(\mathrm{CO}, \mathrm{CH})$. Finally, and although it is not the ultimate goal of solar spectroscopy, solar analyses help to test basic molecular data like dissociation energies and transition probabilities.

Acknowledgements. We are much grateful to Willy Nijs (ORB) for his continuous help. N.G. thanks the Belgian Fonds National de la Recherche Scientifique for financial support. 


\section{References}

Anders E., Grevesse N., 1989, Geochim. Cosmochim. Acta , 53, 197

Bartoe J.-D., Brueckner G.E., Nicolas K.R., Sandlin G.D., Van Hoosier M.E., Jordan C., 1979, MNRAS, 187, 463

Bauschlicher C.W., Langhoff S.R., Taylor P.R., 1988, Astrophys. J., 332, 531

Biémont E., Hibbert A., Godefroid M., Vaeck N., Fawcett B.C., 1991, Astrophys. J., 375, 818

Blatherwick R.D., Murcray F.J., Murcray F.H., Goldman A., Murcray D.G., 1983, Atlas of South Pole IR Solar Spectra, Dept. of Physics, Univ. of Denver

Blomme R., Sauval A.J., Grevesse N., 1993, in "Infrared Solar Physics", eds. D. Rabin, J.T. Jefferies and C. Lindsey, Kluwer, Dordrecht (in press)

Bohn H.U., Wolf B.E., 1984, Astron. Astrophys., 130, 202

Brekke P., 1993, An Ultraviolet Spectral Atlas of the Sun Between 1190 and 1730 A, Astrophys. J. Suppl. 87 ,1 (July, 1993)

Carlson T.A., Duric N., Erman P., Larsson M., 1978, J. Phys. B., 11, 3667

Carroll P.K., McCormack P., O'Connor S., 1976, Astrophys. J., 208, 903

Cartwright D.C., Hay P.J., 1982, Astrophys. J., 257, 383

Chase M.W., Davies C.A., Downey J.R., Frurip D.J., McDonald R.A., Syverud A.N., 1985, JANAF Thermochemical Tables, 3d ed. J. Phys. Chem. Ref. Data 14, Sup.1

Conley C., Halpern J.B., Wood J., Vaughn C., Jackson W.M., 1980, Chem. Phys. Lett. 73,224

Costes M., Naulin C., Dorthe G., 1990, Astron. Astrophys., 232, 270

Davis S.P., Shortenhaus D., Stark G., Engleman R. Jr., Phillips J.G., Hubbard R.P., 1986, Astrophys. J., 303,892 and 307,414

de Jager C., 1959, in Handbuch der Physik, ed. S. Flügge, Vol. LII : Astrophysics III : the Solar System, Springer Verlag, p. 80

Delbouille L., Roland G., Neven L., 1973, Photometric Atlas of the Solar Spectrum from 3000 to $10000 \AA$, Institut d'Astrophysique, Université de Liège

Delbouille L., Roland G., Brault J.W., Testerman L., 1981, Photometric Atlas of the Solar Spectrum from 1850 to $10000 \mathrm{~cm}^{-1}$, Kitt Peak National Observatory, Tucson

Dravins D., Larsson B., Nordlund A., 1986, Astron. Astrophys., 158, 83

Dravins D., Lindegren L., Nordlund A., 1981, Astron. Astrophys., 96, 345

Eres D., Gurnick M., Mc Donald J.D., 1984, J. Chem. Phys. 81, 5552

Farmer C.B., Norton R.H., 1989, A High-Resolution Atlas of the Infrared Spectrum of the Sun and the Earth Atmosphere from Space, Vol. 1, The Sun, NASA Ref. Publ. 1224, Washington

Farrenq R., Guelachvili G., Sauval A.J., Grevesse N., Farmer C.B., 1991, J. Mol. Spectrosc. 149,375

Geller M., 1992, A High-Resolution Atlas of the Infrared Spectrum of the Sun and the Earth Atmosphere from Space, Vol. III, Key to Identification of Solar Features, NASA Ref. Publ. 1224, Washington

Geller M., Sauval A.J., Grevesse N., Farmer C.B., Norton R.H., 1991, Astron. Astrophys. 249, 550

Goldman A., Blatherwick R.D., Murcray F.J., Van Allen J.W., Murcray F.H., Murcray D.G., 1987, New Atlas of Stratospheric IR Absorption Spectra, Vol. I : Line positions and and Identifications; Vol. II : The Spectra, Dept. of Physics, Univ. of Denver

Goldman A., Blatherwick R.D., Kosters J.J., Murcray F.J., Murcray F.H., Murcray D.G., 1992, Atlas of Very High Resolution Stratospheric IR Absorption Spectra, Dept. of Physics, Univ. of Denver 
Grevesse N., Noels A., 1993, in "Origin and Evolution of the Elements", eds. N. Prantzos, E. Vangioni-Flam, M. Cassé, Cambridge University Press, in press

Grevesse N., Sauval A.J., 1970, Astron. Astrophys. 9, 232

Grevesse N., Sauval A.J., 1971a, J. Quant. Spectrosc. Radiat. Transfer 11, 65

Grevesse N., Sauval A.J., 1971b, Astron. Astrophys. 14, 477

Grevesse N., Sauval A.J., 1991, in "The Infrared Spectral Region of Stars", eds. C. Jaschek and Y. Andrillat, Cambridge University Press, p. 215

Grevesse N., Sauval A.J., 1992, Rev. Mexicana Astron. Astrof. 23, 71

Grevesse N., Noels A., Sauval A.J., 1992, in "First SOHO Workshop : Coronal Streamers, Coronal Loops and Coronal and Solar Wind Composition", ESA SP-348, p.305

Grevesse N., Sauval A.J., Blomme R., 1993, in "Infrared Solar Physics", eds. D. Rabin, J.T. Jefferies and C. Lindsey, Kluwer, Dordrecht (in press)

Grevesse N., Sauval A.J., van Dishoeck E.F., 1984, Astron. Astrophys., 141, 10

Grevesse N., Lambert D.L., Sauval A.J., van Dishoeck E.F., Farmer C.B., Norton R.H., 1990 Astron. Astrophys., 232, 225

Grevesse N., Lambert D.L., Sauval A.J., van Dishoeck E.F., Farmer C.B., Norton R.H., 1991 Astron. Astrophys., 242, 488

Gurvich L.V. et al., 1978-1981, Termodynamicheskie Svoistva Individual' nikh Veschev, Vol. 1-3, Eds Moscow: Soviet Acad. Sci.

Hall D.N.W., Noyes R.W., 1969, Astrophys. J. Letters, 4, 143

Hall D.N.W., Noyes R.W., 1972, Astrophys. J. 175, L95

Harris M.J., Lambert D.L., Goldman A., 1987, MNRAS 224, 237

Hibbert A., Biémont E., Godefroid M., Vaeck N. 1991, Astron. Astrophys. Suppl., 88, 505; J. Phys. B. 24, 3943

Hibbert A., Biémont E., Godefroid M., Vaeck N. 1993, Astron. Astrophys. Suppl., 99, 179

Hinkle K. H., Lambert D.L., 1975, MNRAS 170, 447

Hishikawa A., Karawajczyk A., 1993, J. Mol. Spectrosc. 158, 479

Holweger H., Müller E.A., 1974, Solar Physics 39, 19

Huang Y., Barts S.A., Halpern J.B., 1992, J. Phys. Chem. 96, 425

Iglesias C.A., Rogers F.J., Wilson B.G., 1992, Astrophys. J., 397, 717

Irwin A.W., 1981, Astrophys. J. suppl., 45, 621

Irwin A.W., 1987, Astron. Astrophys., 182, 348

Irwin A.W., 1988, Astron. Astrophys. Suppl., 74, 145

Jackson W.M., Payne W., Halpern J.B., Tang X., 1982, Proc. Intern. Conf. on Lasers, p. 72

Jørgensen U.G., Larsson M., 1990, Astron. Astrophys., 238 , 424

Kiselman D., 1991, Astron. Astrophys., 245, L9

Knowles P.J., Werner H.-J., Hay P.J., Cartwright D.C., 1988, J. Chem. Phys. 89 , 7334

Kotlar A.J., Field R.W., Steinfeld J.I., Coxon J.A. 1980, J. Mol. Spectrosc. 80, 86

Kurucz R.L., 1991, in Solar Interior and Atmosphere, eds A.N. Cox, W.C. Livingston, M.S.Matthews, The Univ. of Arizona Press, Tucson, p. 663

Kurucz R.L., 1992, Rev. Mexicana Astron. Astrof. 23, 181

Kurucz R.L., Furenlid I., Brault J.W., Testerman L., 1984, Solar Flux Atlas from 296 to $1300 \mathrm{~nm}$, National Solar Observatory, Tucson, Atlas Nr. 1

Lambert D.L., 1978, MNRAS 182, 249

Lambert D.L., 1988, Publ. Astron. Soc. Pacif. 100, 1202

Lambert D.L., Mallia E.A., 1970, MNRAS 148, 313

Lambert D.L., Gustafsson B., Eriksson K., Hinkle K.H., 1986, Astrophys. J. 62,373 
Larsson M., Siegbahn P.E.M., 1983, Chem. Phys. 76,175

Larsson M., Siegbahn P.E.M., Agren H., 1983, Astrophys. J. 272 , 369

Lavendy H., Gandara G., Robbe J.M., 1984, J. Mol. Spectrosc. 106 , 395

Livingston W., Wallace L., 1991, An Atlas of the Solar Spectrum in the Infrared from 1850 to $9000 \mathrm{~cm}^{-1}$ (1.1 to $\left.5.4 \mu \mathrm{m}\right)$, Technical Report 91-001, National Solar Observatory, Tucson

Lu R., Huang Y., Halpern J.B., 1992, Astrophys. J., 395, 710

Mahan B.H., O'Keefe A., 1981, Astrophys. J. 248, 1209

Maltby P., Avrett E.H., Carlsson M., Kjeldseth-Moe O., Kurucz R.L., Loeser R., 1986, Astrophys. J. 306, 284

Mélen F., Grevesse N., Sauval A.J., Farmer C.B., Norton R.H., Bredohl H., Dubois I., 1989, J. Mol. Spectrosc. 134, 305

Moore C.E., Minnaert M.G.J., Houtgast J., 1966, The Solar Spectrum $2935 \AA$ to 8770 $\AA$, National Bureau of Standards Monograph 61

Moore C.E., Tousey R., Brown C.M., 1982, The Solar Spectrum 3069 - 2095 A, Naval Research Laboratory, NRL Report 8653

Nadeau D., 1988,Astrophys. J., 325, 480

Rao V.M., Rao M.L.P. Rao, P.T., 1982, Phys. Rev. 26 , 1765

Rossi S.C.F., Maciel W.J., 1983, Astrophys. Space Sci., 96, 205

Rossi S.C.F., Maciel W.J., Benevides-Soares, 1985, Astron. Astrophy., 148, 93

Sandlin G.D., Bartoe J.-D.F., Brueckner G.E., Tousey R., VanHoosier M.E., 1986, Astrophys. J. Suppl., 61, 801

Sauval A.J., 1969, Solar Physics 10, 319

Sauval A.J., Tatum J. B., 1984, Astrophys. J. Suppl., 56, 193

Sauval A.J., Blomme R., Grevesse N., 1993 (in preparation)

Sauval A.J., Farrenq R., Guelachvili G., Grevesse N., Farmer C.B., Norton R.H., 1992, Astron. Astrophys., 265, 355

Sauval A.J., Grevesse N., Brault J.W., Stokes G.M., Zander R., 1984, Astrophys. J., 282, 330

Scalo J.M., Ross J.E., 1976, Astron. Astrophys., 48, 219

Seaton M.J., Zeippen C.J., Tully J.A., Pradham A.K., Mendoza C., Hibbert A., Berrington K.A., 1992, Rev. Mexicana Astron. Astrof. 23, 19

Sinha K., Tripathi B.M., 1986, Bull. Astron. Soc. India 14, 40

Smith W.H., 1971, J. Chem. Phys. 54, 1384

Sneden C., Lambert D.L., 1982, Astrophys. J., 259, 381

Swensson J.W., Benedict W.S., Delbouille L., Roland G., 1970, The Solar Spectrum from $\lambda 7498$ to $\lambda 12016$ - A table of Measures and Identifications, Mém. Soc. Roy. Sci. Liège 5

Taherian M.R., Slanger T.G., 1984, J. Chem. Phys. 81,3814

Tatum J.B., 1988, Observatory 108, 55

Tsuji T., 1973, Astron. Astrophys., 23, 411

Vernazza J.E., Avrett E.H., Loeser R., 1976, Astrophys. J. Suppl., 30, 1

Wallace L., Hinckle K., Livingston W., 1993, An Atlas of the Photospheric spectrum from 8900 to $13600 \mathrm{~cm}^{-1}$ (7350 to $\left.11230 A\right)$, Technical Report 93-001, National Solar Observatory, Tucson

Wannenmacher E.A.J., Lin H., Jackson W.M., 1990, J. Phys. Chem. 94, 6608

Wing R.F., Cohen J., Brault J.W., 1977, Astrophys. J., 216, 659

Wöhl H., Engvold O., Brault J.W., 1983, Inst. Theoret. Astrophys. Oslo Rep. 56 